\begin{tabular}{cc}
\hline Revista de & Journal of Integrated \\
GESTÃO COSTEIRA Integrada COSTAL ZONE MANAGEMENT \\
\hline
\end{tabular}

\title{
Vulnerabilidade costeira e perda de ambientes devido à elevação do nível do mar no litoral sul do Rio Grande do Sul *
}

\author{
Yana Friedrich Germani ${ }^{\circledR,}$ a,; Salette Amaral de Figueiredo \\ Lauro Júlio Calliari ${ }^{\text {b }}$ Carlos Roney Armanini Tagliani ${ }^{\text {b }}$
}

\begin{abstract}
Resumo
No atual cenário de mudanças climáticas globais, em que alguns dos efeitos observados são a elevação do nível médio do mar, e o aumento na frequência e intensidade de eventos extremos, regiões costeiras de todo o mundo deverão tornar-se, de forma geral mais vulneráveis. Neste contexto, a costa do Rio Grande do Sul, devido as suas características morfodinâmicas intrínsecas, tais como o alto grau de exposição à dinâmica oceânica e baixa declividade, coloca os vários ecossistemas a ela associados, como praia, dunas, marismas, campos, banhados e sangradouros, numa posição mais vulnerável às variações do nível médio do mar. Visando a um planejamento mais adequado destas áreas no cenário de mudanças climáticas globais, e a fim de determinar uma possível perda de ambientes, no caso de uma elevação do nível do mar em longo prazo (cenário 2100 ), os ambientes costeiros foram inicialmente classificados através da utilização do programa IDRISI, onde foram calculados os percentuais relativos de perda de cada um, para três possíveis cenários de linha de costa, projetados para 2100. Com relação aos resultados obtidos nos cálculos de perda ambiental, considerando as linhas de costa projetadas para 2100, percebemos que todos os ambientes teriam um percentual de área perdida, mesmo no melhor cenário considerado. Neste mesmo cenário onde a linha de costa projetada para 2100 encontra-se recuada $561 \mathrm{~m}$ da posição atual e tem $70 \%$ de probabilidade de ser ultrapassada, observamos também, o maior percentual relativo de perda de área urbana. Para o cenário considerado como intermediário, onde a linha de costa projetada encontra-se recuada $695 \mathrm{~m}$ da atual, e tem $50 \%$ de probabilidade de ser ultrapassada, dunas representam a maior parte da área ambiental perdida, porém, dividem espaço com áreas de campos e em menor escala, áreas de matas. Já, para o pior cenário considerado, onde a linha de costa projetada encontra-se recuada $1032 \mathrm{~m}$ da atual e com $10 \%$ de probabilidade de ser ultrapassada, houve uma inversão na perda ambiental com relação ao cenário anterior. Neste cenário, campos litorâneos e dunas aproximaram-se de $50 \%$ e $35 \%$ de perda ambiental, respectivamente, representando as maiores áreas impactadas. A vulnerabilidade costeira também foi avaliada através do cálculo do Índice de Vulnerabilidade Costeira (IVC), para os cenários atual e futuro (projetado para 2100). Com relação à vulnerabilidade costeira, de forma geral, os valores do IVC calculados para a região foram de 14,4 (atual) e 51,03 (futuro), o que faria com que a mesma deixasse de ser caracterizada como uma região moderadamente vulnerável, e passasse a ser considerada como uma região costeira de vulnerabilidade muito alta à elevação do nível do mar. Dessa forma, as informações aqui obtidas são úteis para planejar adequadamente a ocupação, o uso e a adaptação desta região costeira, bem como, possibilitar estudos futuros acerca do manejo dos ambientes nela encontrados, face ao atual cenário de mudanças climáticas globais.
\end{abstract}

Palavras-chave: Mudanças Climáticas Globais, Índice de Vulnerabilidade Costeira (IVC), Rio Grande do Sul.

\footnotetext{
(a) Corresponding author, to whom correspondence should be addressed.

a Nav Oceanografia Ambiental Ltda. Rua Gildásio Amado,55/ 50, CEP 22631-020, Barra da Tijuca, Rio de Janeiro, RJ, Brasil. e-mail: <yanafg@hotmail.com>

${ }^{\mathrm{b}}$ Universidade Federal do Rio Grande (FURG), Laboratório de Oceanografia Geológica, Caixa Postal 474, CEP: 96201-900, Rio Grande, RS, Brasil. e-mails: Figueiredo <saletteoc@gmail.com>; Calliari <lcalliari@log.furg.br>; Tagliani <ctagliani@log.furg.br>
}

* Submission: 29 JUL 2014; Peer review: 3 SEP 2014; Revised: 19 SEP 2014; Accepted: 12 FEB 2015; Available on-line: 16 FEB 2015
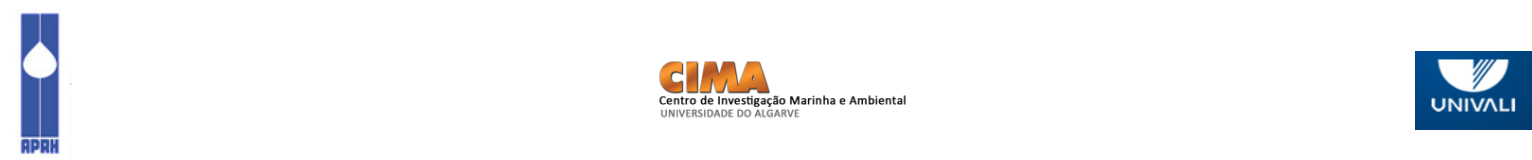


\begin{abstract}
In the current scenario of global climate change, in which some of the observed effects are: the mean sea level rise, and an increased frequency and intensity of extreme events, coastal regions around the world should in general become more vulnerable. In this context, the Rio Grande do Sul coastline, due to its intrinsic morphodynamic characteristics, such as, a low lying coastal gradient combined with a high degree of exposure to ocean dynamics, places several associated ecosystems, such as beach, dunes, marshes, fields, wetlands and washouts in a vulnerable position to changes in sea level. Model simulations of coastal response to sea level rise for the Rio Grande do Sul pointed out Cassino beach sector as being the most affected with the largest shoreline recession along the entire coast. Aiming for adequate planning of these areas under global climate change scenarios, and in order to determine the possible loss of habitats in case of a rise of mean sea level in the long term (2100), present day shoreline was digitalized at the foredune toe limit using in ArcGis, and coastal environments were initially classified using IDRISI program. In this environment, the percentages for each habitat loss were calculated for three possible coastline scenarios, designed for 2100. It was noticed that all environments would display some lost area, even at the best case scenario. In this scenario where the projected shoreline is located at $561 \mathrm{~m}$ inland form the present day shoreline with $70 \%$ probability of being exceeded by 2100, more than half of the dune area would be lost, followed by a smaller fraction of fields. It can also be observed that in this scenario the urban area had the highest percentage loss. For the intermediate scenario where the projected shoreline is located at $695 \mathrm{~m}$ inland from the present day shoreline with $50 \%$ probability of being exceeded, dunes represent the largest loss in habitat, sharing space, with surrounding green fields and to a lesser extent, to forests. Yet for the worst case scenario where the projected shoreline is located at $1032 \mathrm{~m}$ inland from the present day shoreline with $10 \%$ probability of being exceeded, there was a reversal in habitat loss with respect to the intermediate scenario. In this scenario, green fields and coastal dunes approached $50 \%$ and $35 \%$ loss in habitat, respectively, representing the largest impacted areas. It should be highlighted that the fresh water creeks, called washouts, in the best case scenario had its entire area lost. However, in the long term they probably should re-establish its course if topography allows. On the one hand, considering any of the simulated scenarios, as the shoreline retreats the salt marsh area would become closer to the foredune areas. On the other hand, even though not simulated here, there is also the possibility that with sea-level rise it could be inundated through the connecting channel to Patos Lagoon resulting in an increased habitat area. Coastal vulnerability was evaluated through the use of Coastal Vulnerability Index (CVI) for the present and future scenarios for 2100. Looking at coastal vulnerability in general, calculated CVI values for the study area were 14.4 and 51.03, designed for the present and for the year 2100, respectively. The results indicate that a region currently characterized as a moderately vulnerable, should become highly vulnerable in the future due to the rising of mean sea level as projected. This change would take place basically influenced by two of the index variables: shoreline erosion/accretion rates; and relative sea-level change, considered here as the controlling variables for coastal vulnerability under climate change in this area. Thus, the information obtained is critical to properly plan, land use and adaptation of this coastal region under the current scenario for global climate change, as well as enable future studies on habitat management.
\end{abstract}

Keywords: Global Climate Changes, Coastal Vulnerability Index (CVI), Rio Grande do Sul.

\section{Introdução}

Atualmente vivemos em um período marcado por mudanças climáticas globais, o qual vem sendo estudado com especial interesse pela comunidade científica que se dedica às ciências da natureza e da sociedade. Alterações climáticas são alguns dos efeitos já visíveis da grande modificação que o planeta está sofrendo devido ao fenômeno do aquecimento global (Andrade \& Lacerda, 2009; Bindoff et al., 2007). Outros efeitos observados, são a acentuação do efeito El Niño, catástrofes de grandes proporções, desequilíbrio das chuvas e grandes enchentes.

A zona costeira que se destaca pelos seus recursos naturais e diversidade ambiental, bem como pelo seu grande potencial para o desenvolvimento de atividades econômicas múltiplas (Carter, 1990; Diehl et al., 2010) poderá ter os efeitos das mudanças climáticas agravados pela intensa antropização dos espaços urbanos, trazendo situações que caracterizam vulnerabilidades e adaptações para as populações e instalações (IPCC, 2001).

Resultados obtidos a partir de modelagem (Meehl et al., 2007; Marengo, 2006), em escalas que vão de interanu- al até decadal, e a longo prazo, constataram que a intensidade de tempestades tropicais e extratropicais aumentarão, o que implica em mais impactos na costa do que apenas aos atribuídos a elevação do nível médio do mar, especialmente para sistemas costeiros tropicais e de médias latitudes. Assim sendo, a região costeira do Estado do Rio Grande do Sul, por ser uma extensa planície arenosa e de baixo gradiente topográfico, ficará ainda mais vulnerável a eventos erosivos com o aumento do nível médio do mar, quando comparada a áreas mais íngremes, especialmente durante eventos extremos.

Somente na década de 90, o enfoque nas possíveis alterações da linha de costa e seus efeitos na erosão costeira, devido a uma elevação do nível médio do mar, despertaram maior atenção dos cientistas, da mídia e do público em geral. Dentre os principais indicadores de tais elevações, destacam-se: o recuo da linha de costa, a erosão costeira, o desaparecimento de ilhas, a destruição de ecossistemas costeiros e áreas urbanizadas, dentre outros. Assim, desde o avanço desses estudos, várias tentativas para prever este comportamento durante os próximos séculos têm surgido. 
No que se refere à média das mudanças do nível médio do mar feitas no Brasil, os dados sobre o comportamento atual do nível médio do mar possuem disponibilidade limitada, o que, eleva a incerteza para os modelos de resposta costeira para o futuro aumento do nível do mar. No Brasil, poucos locais apresentam registros suficientes para permitir comparações climáticas (Mesquita, 2003). Apenas no ano de 2004 houve a implantação do Sistema Global de Observação do Nível do Mar (GLOSS), coordenado pelo Centro de Hidrografia da Marinha do Brasil (CHM). O principal objetivo do projeto é unir esforços de instituições brasileiras que dependem das observações do nível do mar para seus monitoramentos e pesquisas.

Neste trabalho, destacaremos os impactos causados pela elevação do nível médio do mar, ocasionada pelas mudanças climáticas, para a região costeira adjacente a desembocadura da Lagoa dos Patos. Assim considerando as características geológicas e morfodinâmicas, principalmente o caráter oceânico aberto; a orientação Sudoeste-Nordeste da linha de costa, que a expõe às tempestades mais intensas provenientes do quadrante SulSudeste; e o baixo gradiente topográfico da antepraia; a costa gaúcha -atualmente inserida numa faixa de alto risco a qualquer elevação do nível do mar, ficará nos cenários de mudanças climáticas globais relatadas no Ipcc (2007) e Church et al. (2013), ainda mais vulnerável à erosão.

Projeções de elevação do nível médio do mar apresentadas no terceiro e quarto relatórios de avaliação no Painel Intergovernamental sobre Mudanças Climáticas (IPCC, 2001; IPCC, 2007) mostram taxas similares de aumento do nível do mar, no entanto, este último depende de um maior número de modelos climáticos de crescente complexidade e realismo, resultando em taxas mais confiáveis de aumento do nível do mar global que variam entre $0,18 \mathrm{~m}$ a $0,59 \mathrm{~m}$ em 2100 . Com base nos resultados das projeções de elevação acelerada do nível médio do mar, foram desenvolvidos modelos capazes de prever a resposta costeira em diferentes situações de comportamento do nível médio do mar.

Historicamente, a Praia do Cassino vem sofrendo acreção (Lélis \& Calliari, 2004) devido, em parte, à construção dos molhes da Barra do Rio Grande, a qual interferiu no balanço sedimentar da área ao obstruir o transporte de sedimentos para Nordeste, acarretando em um aumento localizado na largura da praia na região do Cassino. Entretanto, Figueiredo (2013), ao simular cenários futuros de resposta costeira a elevação do nível do mar para a costa no Rio Grande do Sul, utilizando o STM (Shoreline Translation Model) de Cowell et al. (1992), constatou os maiores valores de recuo da linha de costa para o setor que inclui a Praia do Cassino alcançando distâncias médias de recuo de até $695 \mathrm{~m}$. A autora utiliza uma faixa de valores de elevação do nível do mar com valor mínimo de $0,18 \mathrm{~m}$, modal de $0,89 \mathrm{~m}$ e máximo de $1,1 \mathrm{~m}$ para o ano de 2100 . Estes são valores combinados de projeções de Rahmstorf (2007) e do Ipcc (2007). Devido ao fato de que a incerteza na própria modelagem de clima é inerente a todas as previsões e está intimamente relacionada o grau de contribuição antropogênica e em relação à dinâmica do derretimento das placas de gelo (IPCC, 2007), os resultados da modelagem de resposta costeira sob condições de mudanças climáticas é apresentado na forma de probabilidade de serem excedidas, e.g., a linha de costa localizada a $695 \mathrm{~m}$ da linha de costa atual tem $50 \%$ de probabilidade de ser ultrapassada em 2100.

Neste trabalho, as projeções de linha de costa obtidas por Figueiredo (2013) para o ano de 2100, são resultantes da aplicação do modelo proposto por Cowell et al. (2006), onde os impactos relacionados aos efeitos das mudanças climáticas incluem aumento do nível médio do mar, alterações no clima de ondas e distúrbios no balanço de sedimentos ao longo da costa, podendo resultar em erosão e recuo da mesma. O autor também considera que, áreas mais vulneráveis à erosão costeira podem ser identificadas em termos de distância continente adentro a partir da atual linha de dunas, à medida que a linha de costa recua num determinado período de tempo no futuro (estabelecido na modelagem). $\mathrm{O}$ recuo total em função do tempo leva em consideração os seguintes termos: tendência média de recuo devido ao desequilíbrio no suprimento de sedimentos litorâneos (no caso de balanço negativo), tendência média de recuo devido à elevação acelerada do nível do mar, e aumento da erosão devido à ocorrência de tempestades (ver Eq. 1). Assim, as previsões de recuo da linha de costa podem ser utilizadas para estimar o volume de perda de areia e futuramente prever custos da realimentação artificial necessária para preservar praias e proteger os recursos imediatamente interiores a elas.

$$
R(t)=\overline{R_{V}}(t)+\overline{R_{S L}}(t)+k(t) S_{D}
$$

onde, $R(t)$ é o recuo total, $\overline{\mathbf{R}_{\mathbf{V}}}(\mathrm{t})$ é o recuo médio devido ao desequilíbrio do suprimento sedimentar, $\overline{R_{S L}}(t)$ é o recuo médio devido à elevação acelerada do nível do mar e $k(t) S_{D}$ é a alteração do balanço sedimentar devido ao aumento da intensidade das tempestades.

Também, a fim de prever os impactos da elevação do nível do mar, uma metodologia para identificar as áreas que podem ser mais vulneráveis a essa elevação no futuro foi desenvolvida (Gornitz, 1991). Esta técnica será descrita com detalhes na subseção 3.2.

Transgressões e regressões marinhas ocorreram anteriormente durante o Pleistoceno e Holoceno moldando a costa como a conhecemos hoje. Estimativas indicam que durante o Holoceno no Rio Grande do Sul, o nível médio do mar atingiu altitudes máximas entre 2 e $4 \mathrm{~m}$ 
acima do nível atual (Dillenburg et al., 2000; Dillenburg et al., 2005). Entretanto, os impactos de uma subida acelerada do nível do mar na presente configuração costeira, ainda são em grande parte desconhecidos.

\section{2. Área de estudo}

A costa do Rio Grande do Sul (RS), de orientação sudoeste-nordeste (Fig. 1), possui características oceânicas, devido à ação de ondas, correntes litorâneas e o vento, que são os principais fatores responsáveis por alterar a sua morfologia. A plataforma continental adjacente é larga, com 150 a $200 \mathrm{~km}$ de extensão, apresentando profundidades máximas variando entre $100 \mathrm{e}$ $140 \mathrm{~m}$, e declividade suave da ordem de 0,5 a $1,5 \mathrm{~m} / \mathrm{km}$ (Dillenburg et al., 2000). Ao longo dos $640 \mathrm{~km}$ do litoral do estado observam-se poucas descargas flúviolagunares importantes (Figueiredo \& Calliari, 2005), porém, inúmeros cursos de água doce, conhecidos como sangradouros, estão presentes.

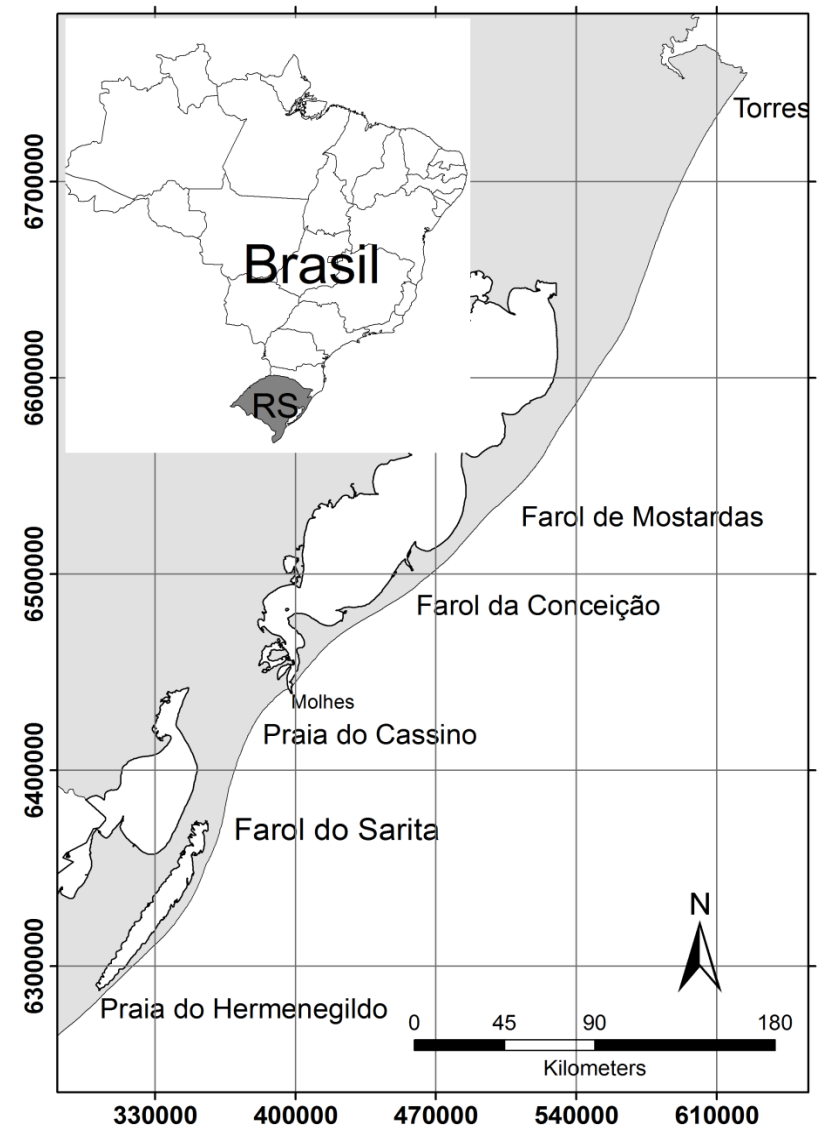

Figura 1 - Localização da área de estudo - Região a partir dos Molhes da Barra de Rio Grande até o Farol do Sarita.

Figure 1 - Location of the study area - Region from Rio Grande jetties to Sarita Lighthouse.

A região é incluída no regime de micromarés, onde as oscilações têm amplitude máxima de $0,5 \mathrm{~m}$. As maiores alterações do nível médio do mar percebidas nesse local devem-se às chamadas marés meteorológicas ou de tempestades. Já as ondulações que atingem a região costeira da área de estudo têm duas direções predomi- nantes: de leste-nordeste e de sudeste (Martinho et al., 2009; Pianca et al., 2010).

A ação morfogênica na área estudada, relacionada com a atividade das correntes, é controlada basicamente pelas correntes litorâneas induzidas pelas ondas e ventos e, muito secundariamente, por correntes fluviais. Do mesmo modo, Fontoura (2013) descreve que o transporte longitudinal na região é bidirecional, apresentando resultante positiva para nordeste.

\section{Material e métodos}

\subsection{Análises de Perda Ambiental}

$\mathrm{O}$ mapeamento das unidades ambientais presentes na área de estudo foi realizado em meio digital, através da técnica de classificação supervisionada (Maxlike) disponível no SIG Idrisi, utilizando imagens do satélite ALOS de 2010, com resolução espacial de 10 metros. A linha de costa atual foi digitalizada no SIG ArcGis seguindo a base das dunas frontais. As seguintes unidades foram identificadas e mapeadas: marismas, dunas, sangradouros, banhados, campos litorâneos, matas e áreas com a infraestrutura urbana.

Figueiredo (2013) estimou através de modelagem o recuo da linha de costa na área de estudo para o ano de 2100 em condições de mudanças climáticas. Suas projeções de linha de costa foram apresentadas na forma de distribuição probabilidade, onde cada valor projetado para futura posição da linha de costa em metros está associado a uma probabilidade (de $0 \%$ a $100 \%$ ) de ser ultrapassado num dado horizonte temporal, neste caso o ano 2100. A partir destas projeções, foram selecionadas para este estudo três linhas de costa projetadas com as respectivas probabilidades de serem ultrapassadas em 2100: linha azul, com $70 \%$ de probabilidade de ser ultrapassada $(1: 1,44)$; linha laranja, $50 \%$ de probabilidade de ser ultrapassada (1:2); linha verde, $10 \%$ de probabilidade (1:10) (Fig. 2). Para cada situação/posição da linha de costa projetada foram calculadas as perdas ambientais (áreas e percentuais relativos) utilizando os métodos overlay e area disponíveis no programa Idrisi.

A Praia do Cassino, no município de Rio Grande, RS, encontra-se ao sul da desembocadura da Laguna dos Patos, a partir da qual se desenvolve uma extensa restinga (barreira arenosa) com $220 \mathrm{~km}$ de extensão, limitada ao sul pelo Arroio Chuí. A área de estudo compreende um embaiamento sutil de aproximadamente $60 \mathrm{~km}$ de extensão (zona UTM 22S, entre 396383/6442021 e 364937/6382475), desde a raiz do Molhe Oeste até a região do Farol do Sarita (Fig. 1). A região possui características semelhantes, representando uma unidade geomorfológica da barreira arenosa holocênica (Tomazelli \& Villwock, 2005) apresentando sistemas praiais dissipativos, campos de dunas, marismas e sangradouros associados, e suas praias são compostas de areia quartzosa de granulometria fina. 


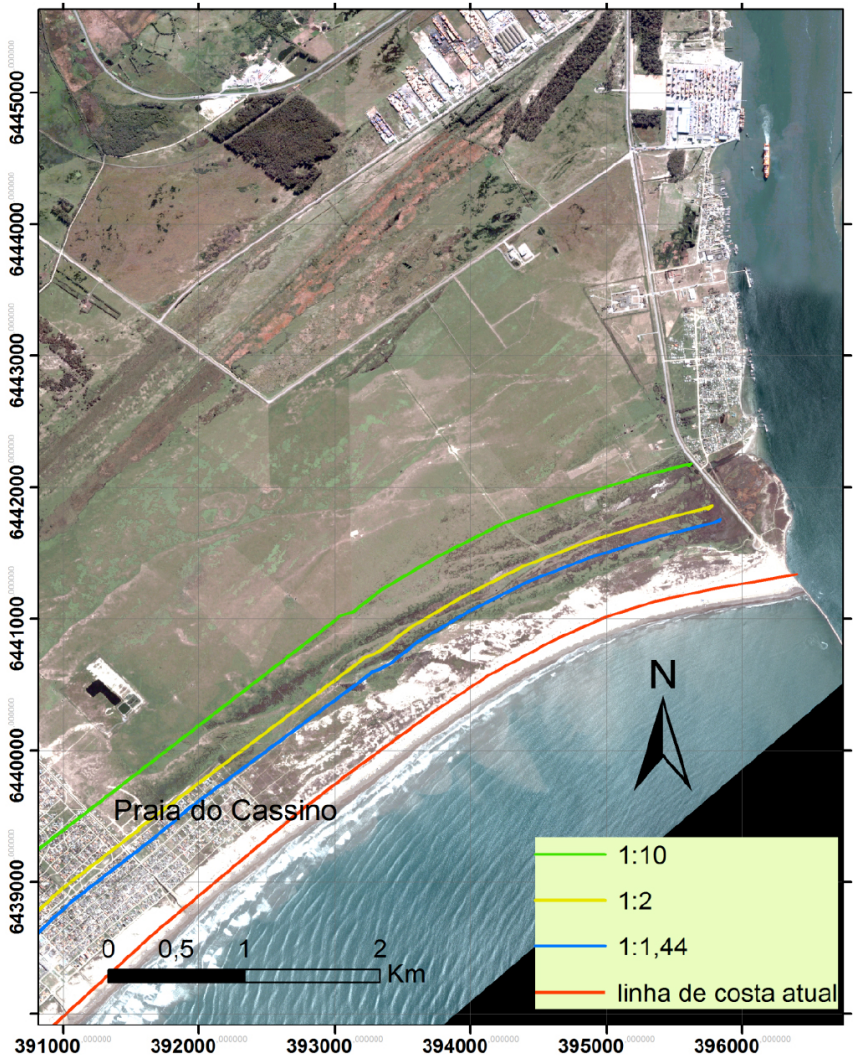

Figura 2 - Contornos representando as possíveis futuras linhas de costa para a Praia do Cassino, com suas respectivas probabilidades de serem ultrapassadas, ou diferentes níveis de risco, para 2100. Adaptado de Figueiredo (2013).

Figure 2 - Contours representing possible future shorelines to Cassino's Beach, with their respective probabilities of exceedence or different risk levels by 2100. Adapted from Figueiredo (2013).

\section{2. Índice de Vulnerabilidade Costeira à Elevação do Nível do Mar}

Para descrever a vulnerabilidade a uma mudança física como o aumento do nível médio do mar na costa em estudo, foi utilizada uma metodologia desenvolvida por Gornitz (1991). Esta técnica utiliza diferentes intervalos de vulnerabilidade (muito baixa a muito alta), associando a cada um deles um valor (Tabela 1).

Valendo-se de seis variáveis (físicas e geológicas), e conhecendo seus valores referentes à área estudada, os valores dos intervalos de vulnerabilidade correspondentes a cada parâmetro são incorporados a uma equação (ver Eq. 2). Esta produz um índice de vulnerabilidade costeira (IVC), onde: valores de IVC abaixo de 8,7 são incluídos na categoria de baixa vulnerabilidade; valores entre 8,7-15,6 são considerados de vulnerabilidade moderada; valores entre 15,6 e 20 indicam alta vulnerabilidade; valores de IVC acima de 20 são classificados como de vulnerabilidade muito alta. A partir dessa técnica, foi calculado o índice para a costa atual, bem como o projetado para o ano de 2100 , e comparados os valores obtidos.

$$
I V C=\sqrt{\frac{\left(A^{*} B^{*} C^{*} D^{*} E^{*} F\right)}{6}}
$$

Onde: $A$ - geomorfologia; $B$ - taxas de erosão/acreção da linha de costa $\left(\mathrm{m}\right.$. ano $\left.^{-1}\right) ; C$ - declividade da costa $(\%)$; $D$ - mudanças relativas do nível do mar $\left(\mathrm{mm}^{\mathrm{anno}}{ }^{-1}\right) ; E$ altura significativa de onda (m); e $F$ - amplitude de maré $(\mathrm{m})$.

Tabela 1 - Valores das variáveis para o cálculo do Índice de Vulnerabilidade para a Costa Atlântica. Fonte: Adaptada de Gornitz (1991).

Table 1 - Variables values for calculating the Vulnerability Index for the Atlantic Coast. Source: Adapted from Gornitz (1991).

\begin{tabular}{c|c|c|c|c|c}
\hline Variáveis & $\begin{array}{c}\text { Muito Baixa } \\
\mathbf{1}\end{array}$ & $\begin{array}{c}\text { Baixa } \\
\mathbf{2}\end{array}$ & $\begin{array}{c}\text { Moderada } \\
\mathbf{3}\end{array}$ & $\begin{array}{c}\text { Alta } \\
\mathbf{4}\end{array}$ \\
\hline Geomorfologia & $\begin{array}{c}\text { Costas de falésia } \\
\text { rochosa, fiord }\end{array}$ & $\begin{array}{c}\text { Falésias médias, } \\
\text { costas recortadas }\end{array}$ & $\begin{array}{c}\text { Falésia baixa, } \\
\text { costas de deriva } \\
\text { glacial, planície } \\
\text { aluvial }\end{array}$ & $\begin{array}{c}\text { Praias cas- } \\
\text { calhosas, } \\
\text { estuários, lagoas }\end{array}$ & $\begin{array}{c}\text { Praias arenosas, } \\
\text { pântanos salgados, } \\
\text { planos lamosos, } \\
\text { deltas, mangues, } \\
\text { recifes de coral }\end{array}$ \\
\hline $\begin{array}{c}\text { Taxas de erosão / } \\
\text { acreção (m/a) }\end{array}$ & $>2,0$ & $1,0-2,0$ & $-1,0-1,0$ & $-2,0--1,0$ & $<2,0$ \\
\hline $\begin{array}{c}\text { Declividade da } \\
\text { costa (\%) }\end{array}$ & $>1,20$ & $1,20-0,90$ & $0,90-0,60$ & $0,60-0,30$ & $<0,30$ \\
\hline $\begin{array}{c}\text { Variação relativa } \\
\text { do nível médio do } \\
\text { mar (mm/a) }\end{array}$ & $<1,8$ & $1,8-2,5$ & $2,5-3,0$ & $3,0-3,4$ & $>3,4$ \\
\hline $\begin{array}{c}\text { Altura signi- } \\
\text { ficativa da onda } \\
\text { (m) }\end{array}$ & $<0,55$ & $0,55-0,85$ & $0,85-1,05$ & $1,05-1,25$ & $>1,25$ \\
\hline $\begin{array}{c}\text { Amplitude da } \\
\text { maré (m) }\end{array}$ & $>6,0$ & $4,0-6,0$ & $2,0-4,0$ & $1,0-2,0$ & $<1,0$ \\
\hline
\end{tabular}




\section{Resultados e discussão}

\subsection{Perda ambiental para 2100}

Nas Tabelas 2, 3 e 4 são apresentados os resultados dos cálculos de perda ambiental devido à elevação do nível do mar, em $\mathrm{km}^{2}$ e $\%$ relativo. Foi considerada como máxima área perdida, apenas a contida entre a linha da base das dunas frontais e a linha de costa modelada com $10 \%$ de probabilidade de ser ultrapassada (recuada $1032 \mathrm{~m}$ da atual linha de costa). Entretanto, para fins de classificação inicial dos ambientes, foi incluída toda a área da barreira arenosa holocênica (Fig. 3). Para um melhor entendimento, os resultados obtidos foram separados em três cenários.

\section{1) Melhor cenário}

Este cenário utiliza a linha de costa modelada que recuou $561 \mathrm{~m}$ da posição da atual linha de costa e tem $70 \%$ de chance de ser ultrapassada até o ano 2100. Nesta situação, observa-se que mais da metade da área perdida é representada por dunas, seguida de uma fração menor, porém significativa, de campos (Tabela 2). Comparando os três cenários (Tabelas 2, 3 e 4), percebe-se com este cenário o maior percentual de perda de área urbana, além da totalidade da área de sangradouros, já que estes se limitam à região mais próxima à costa.

Tabela 2 - Área perdida e respectivo percentual relativo para cada ambiente, considerando a possível linha de costa projetada para 2100 , com $70 \%$ de probabilidade de ser ultrapassada.

Table 2 - Lost area and its respective relative percentage for each environment, considering the possible shoreline projected for 2100 , with $70 \%$ probability of being exceeded.

\begin{tabular}{c|c|c}
\hline Ambientes & Área Perdida $\left(\mathbf{k m}^{\mathbf{2}}\right)$ & \% relativo \\
\hline Dunas & 20,71 & 55,40 \\
\hline Matas & 1,39 & 3,72 \\
\hline Banhados & 1,04 & 2,78 \\
\hline Campos & 11,70 & 31,3 \\
\hline Marismas & 0,08 & 0,21 \\
\hline Sangradouros & 0,12 & 0,32 \\
\hline Área Urbana & 2,34 & 6,26 \\
\hline TOTAL & 37,38 & $\cong 100$ \\
\hline
\end{tabular}

\section{2) Cenário intermediário}

Este cenário utiliza a linha de costa modelada que recuou $695 \mathrm{~m}$ da posição da atual linha de costa e tem $50 \%$ de chance de ser ultrapassada até o ano 2100 . Na Tabela 3 observa-se que, do mesmo modo que no contexto anterior, as dunas ainda representam a maior parte de área ambiental perdida. Porém, dividindo espaço com áreas de campos e, em menor escala, de matas.

Tabela 3 - Área perdida e respectivo percentual relativo para cada ambiente, considerando a possível linha de costa projetada para 2100 , com $50 \%$ de probabilidade de ser ultrapassada.

Table 3 - Lost area and its respective relative percentage for each environment, considering the possible shoreline projected for 2100, with $50 \%$ probability of being exceeded.

\begin{tabular}{c|c|c}
\hline Ambientes & Área Perdida $\left(\mathbf{k m}^{2}\right)$ & \% relativo \\
\hline Dunas & 21,31 & 46,93 \\
\hline Matas & 3,07 & 6,76 \\
\hline Banhados & 1,45 & 3,19 \\
\hline Campos & 16,6 & 36,56 \\
\hline Marismas & 0,09 & 0,20 \\
\hline Sangradouros & 0,12 & 0,26 \\
\hline Área Urbana & 2,77 & 6,10 \\
\hline TOTAL & 45,41 & $\cong 100$ \\
\hline
\end{tabular}

\section{3) Pior cenário}

Este cenário utiliza a linha de costa modelada que recuou $1032 \mathrm{~m}$ da posição da atual linha de costa e tem $10 \%$ de chance de ser ultrapassada até o ano 2100 . Este cenário mostra uma inversão no percentual dos ambientes perdidos (Tab. 4). Pode-se observar que a área de campos foi a mais afetada com a máxima incursão marinha considerada, chegando próximo a $50 \%$ de perda. Com aproximadamente $35 \%$ de área perdida estão as dunas, e apresentando pouco mais de $10 \%$, as matas.

Tabela 4 - Área perdida e respectivo percentual relativo para cada ambiente, considerando a possível linha de costa projetada para 2100 , com $10 \%$ de probabilidade de ser ultrapassada.

Table 4 - Lost area and its respective relative percentage for each environment, considering the possible shoreline projected for 2100 , with $10 \%$ probability of being exceeded.

\begin{tabular}{c|c|c}
\hline Ambientes & Área Perdida $\left(\mathbf{k m}^{\mathbf{2}}\right)$ & \% relativo \\
\hline Dunas & 23,61 & 34,21 \\
\hline Matas & 7,08 & 10,26 \\
\hline Banhados & 2,25 & 3,26 \\
\hline Campos & 31,94 & 46,28 \\
\hline Marismas & 0,14 & 0,20 \\
\hline Sangradouros & 0,12 & 0,17 \\
\hline Área Urbana & 3,87 & 5,61 \\
\hline TOTAL & 69,01 & $\cong 100$ \\
\hline
\end{tabular}




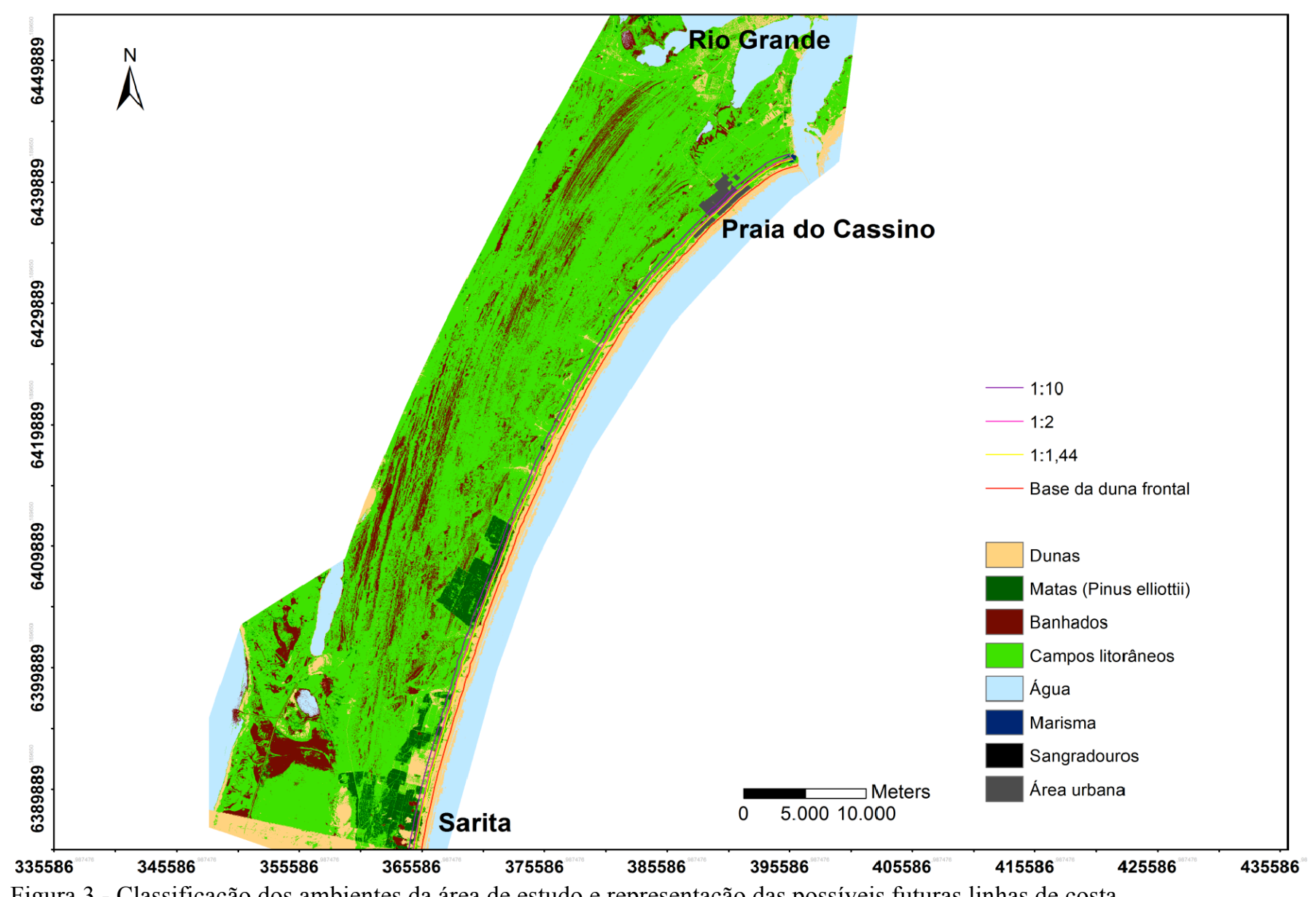

Figura 3 - Classificação dos ambientes da área de estudo e representação das possíveis futuras linhas de costa.

Figure 3 - Environmental classification of the study area and representation of possible future shorelines.

Os resultados aqui apresentados assumem um cenário de subida acelerada de nível do mar numa região costeira de gradiente muito suave, com declividade da antepraia em torno de $0,08^{\circ}$ e dunas que não ultrapassam 3,5 m.

Entretanto, devemos ter em mente que a reorganização morfodinâmica em larga escala temporal, de dezenas a centenas de anos se dá de forma lenta e que não ocorrerá uma inundação abrupta dos ambientes pela subida do nível do mar em longo prazo. Esta reorganização dos ambientes está relacionada com a transladação da linha de costa em função de trocas sedimentares transversais entre a região emersa da barreira, incluindo dunas frontais e áreas posteriores e o limite inferior da antepraia como resultado da subida do nível médio do mar (Bruun, 1988), além de trocas longitudinais que influenciam o balanço sedimentar costeiro no setor como um todo.

Por outro lado, mesmo que ambientes como o pós-praia e campos de dunas migrem gradativamente continente adentro, regiões mais interiorizadas e estáveis, como as marismas, campos litorâneos, banhados e até áreas urbanizadas, ficarão de qualquer forma mais próximas da linha de costa e assim mais vulneráveis aos impactos de eventos extremos. Adicionalmente, teremos impactos sobre as diversas comunidades da fauna e flora da zona costeira.

No caso específico dos sangradouros, mesmo no melhor cenário considerado havendo perda total de área, foi concluído que, em longo prazo eles restabeleceriam sua drenagem em direção ao mar. Isso pode ser comprovado através de observações do interior da barreira arenosa holocênica, onde ficaram evidentes interrupções nos cordões litorâneos que dão origem aos canais. Este fato também foi observado por Hesp et al. (2007) próximo à Praia de Curumim, no litoral norte do RS, onde inúmeros paleo-sangradouros corriam através da barreira holocênica em direção ao mar, estando frequentemente ligados aos canais atualmente ativos. Estes paleosangradouros provavelmente foram formados durante $o$ Holoceno, quando o nível médio do mar na região caía progressivamente, após ter atingido um máximo de elevação por volta de 5100 anos atrás (cerca de $1-3 \mathrm{~m}$ acima do atual), atuando como um sistema organizado de drenagem (Martin et al., 1979).

Com relação à única ocorrência de marisma na área de estudo, restrita às adjacências do Molhe Oeste, observou-se que em todos os cenários possíveis há alguma perda de área deste ambiente. Embora não represente grande significância, em termos de percentual perdido, 
a marisma é um dos ambientes de maior relevância ecológica e sensibilidade ambiental da área, com relação à fauna e flora. Por ser uma região estável, em termos morfodinâmicos, diferentemente da região de dunas, a princípio, este ambiente não se deslocaria continente adentro devido à uma elevação acelerada do nível do mar. Assim, concluímos que, as marismas ficarão, em qualquer um dos três possíveis cenários projetados, localizadas mais próximas dos cordões de dunas do que atualmente. Dessa forma, tornando-se mais vulneráveis aos impactos da transgressão marinha. Conforme Huiskes (1990), um pequeno aumento do nível médio do mar seria suficiente para acarretar mudanças na zonação desse ecossistema, até sua total eliminação.

Por outro lado, foi considerado que, por haver conexão entre o estuário da Lagoa dos Patos e a região de marismas em questão, existe a possibilidade de que haja um aumento de área deste ambiente. Com a subida do nível do mar, o nível da lagoa também deve subir, influenciando no acréscimo da área de marismas.

Segundo Costa (1998), existem cerca de 70 espécies de plantas que habitam a região de marismas no estuário inferior. Estas possuem características muito específicas, com diferentes gradientes de tolerância à inundação, dessecação e salinidade. Assim, em qualquer uma das duas situações, seja de perda ou acréscimo de área, elas sofreriam com o estresse às mudanças da dinâmica ambiental. Dessa forma, poderiam facilmente desaparecer, caso não forem capazes de se adaptar a essas alterações ocasionadas pela elevação do nível médio do mar.

Por fim, o último ambiente a ser considerado neste estudo, também sujeito aos impactos da subida do nível do mar - o Urbano - é o que oferece maior preocupação à população que vive na região costeira. Por afetar diretamente a segurança da vida humana e seus bens materiais, o risco de uma inundação provocando perdas ou danos as suas propriedades mantém as pessoas em estado de alerta. Embora a diferença seja pouco expressiva em nossa área de estudo, o percentual representado pela área urbana é maior quanto mais próximo à costa. Isto representa uma tendência nas regiões costeiras de todo o mundo, tornando os riscos de uma elevação do nível médio do mar ainda mais preocupantes. Foi considerado ainda, que a elevação média do nível do mar, quando associada com tempestades costeiras e ressacas (marés meteorológicas), potencializará ainda mais os impactos previstos para essa área.

Assim sendo, para os futuros cenários projetados, certamente serão necessárias estratégias de adaptação, que podem incluir obras de proteção costeira para conter o avanço marinho, e realocação, mas principalmente um melhor planejamento da ocupação em zonas costeiras vulneráveis aos impactos diretos das mudanças climáticas globais.

\subsection{Vulnerabilidade Costeira à Elevação do Nível do Mar: Cenários Atual e Futuro}

Como forma de quantificar a vulnerabilidade da Praia do Cassino à elevação acelerada do nível do mar, foram inseridos os valores da região, relativos a cada uma das variáveis, físicas e geológicas, na tabela utilizada para o cálculo do Índice de Vulnerabilidade para a Costa Atlântica de Gornitz (1991). Foi obtido um valor de IVC de 14,4 (ver Equação 3), classificando a praia como moderadamente vulnerável a uma elevação do nível do mar na atualidade, conforme mostrado na Tabela 5.

Tabela 5 - Valores dos parâmetros físicos e geológicos para classificação de vulnerabilidade ao aumento do nível do mar para a região de estudo. Taxas de erosão/acreção da linha de costa segundo Lélis \& Calliari (2004); declividade da costa segundo Dillenburg et al. (2000); mudanças relativas do nível do mar segundo Church \& White (2006); altura significativa de onda segundo Pianca et al.(2010); amplitude de maré segundo Calliari \& Klein (1993).

Table 5 - Values of physical and geological parameters for classification of vulnerability to rising sea level to study area. Rates of erosion / accretion of shoreline according to Lelis \& Calliari (2004). Slope of the coast from Dillenburg et al. (2000), relative changes in sea level following Church \& White (2006), significant wave height according to Pianca et al.(2010); tidal range from Calliari \& Klein(1993).

\begin{tabular}{|c|c|c|c|}
\hline Geomorfologia & Parâmetros & $\begin{array}{l}\text { Valores na } \\
\text { classi- } \\
\text { ficação }\end{array}$ & $\begin{array}{l}\text { Vulnera- } \\
\text { bilidade }\end{array}$ \\
\hline $\begin{array}{l}\text { Taxas de erosão / } \\
\text { acreção (m/a) }\end{array}$ & $\begin{array}{c}\text { Praias are- } \\
\text { nosas, } \\
\text { marismas }\end{array}$ & 5 & $\begin{array}{l}\text { Muito } \\
\text { Alta }\end{array}$ \\
\hline $\begin{array}{l}\text { Declividade da } \\
\text { costa }(\%)\end{array}$ & $+3,2$ & 1 & $\begin{array}{l}\text { Muito } \\
\text { Baixa }\end{array}$ \\
\hline $\begin{array}{l}\text { Variação relativa } \\
\text { do nível médio }\end{array}$ & 0,025 & 5 & $\begin{array}{l}\text { Muito } \\
\text { Alta }\end{array}$ \\
\hline $\begin{array}{l}\text { Altura signi- } \\
\text { ficativa da onda }\end{array}$ & 2 & 2 & Baixa \\
\hline $\begin{array}{l}\text { Amplitude da } \\
\text { maré }(\mathrm{m})\end{array}$ & $1-2$ & 5 & $\begin{array}{l}\text { Muito } \\
\text { Alta }\end{array}$ \\
\hline Geomorfologia & 0,5 & 5 & $\begin{array}{l}\text { Muito } \\
\text { Alta }\end{array}$ \\
\hline
\end{tabular}

$\mathrm{IVC}=\sqrt{\frac{\left(5^{*} 1^{*} 5^{*} 2^{*} 5^{*} 5\right)}{6}} \cong 14,4$

Ao observar a Tabela 5, nota-se que, se consideradas constantes as variáveis: geomorfologia, declividade da costa, altura significativa de onda e amplitude de maré; e utilizando os parâmetros das duas variáveis restantes, para o cenário projetado (2100), a região de estudo seria classificada como muito altamente vulnerável a uma 
elevação do nível do mar, com um IVC de 51,03 (ver Equação 4).

Segundo Toldo Jr. et al. (2005), a retração e progradação das zonas costeiras arenosas submetidas ao regime de micromarés, caso da região em estudo, resultam principalmente da dinâmica entre a quantidade e tipo de suprimento sedimentar, energia física de ondas e mudanças relativas do nível médio do mar.

Neste sentido, a Tabela 6 apresenta os valores dos parâmetros físicos e geológicos para classificação de vulnerabilidade ao aumento do nível médio do mar, projetados para 2100 por Figueiredo (2013), enquanto a Equação 4 resulta no IVC calculado para o mesmo cenário futuro.

Tabela 6 - Valores dos parâmetros físicos e geológicos para classificação de vulnerabilidade ao aumento do nível do mar para a região de estudo, para um cenário futuro (2100). Taxas de erosão/acreção da linha de costa, e mudanças relativas do nível do mar, adaptadas de Figueiredo (2013); declividade da costa segundo Dillenburg et al.(2000); altura significativa de onda segundo Pianca et al.(2010); amplitude de maré segundo Calliari \& Klein (1993).

Table 6 - Physical and geological parameters for classification of coastal vulnerability to sea level rise, for a future scenario (2100). Rates of shoreline erosion/accretion and relative changes in sea level, modified from Figueiredo (2013); coastal slope from Dillenburg et al. (2000), significant wave height according to Pianca et al.(2010); tidal range from Calliari \& Klein (1993).

\begin{tabular}{|c|c|c|c|}
\hline Variáveis & Parâmetros & $\begin{array}{l}\text { Valores } \\
\text { na } \\
\text { classi- } \\
\text { ficação }\end{array}$ & $\begin{array}{l}\text { Vulnera- } \\
\text { bilidade }\end{array}$ \\
\hline Geomorfologia & $\begin{array}{l}\text { Praias are- } \\
\text { nosas, } \\
\text { marismas }\end{array}$ & 5 & Muito Alta \\
\hline $\begin{array}{c}\text { Taxas de } \\
\text { Erosão/Acreção } \\
\text { da Linha de } \\
\text { Costa (m/ano) }\end{array}$ & $-11,47$ & 1 & Muito Alta \\
\hline $\begin{array}{c}\text { Declividade da } \\
\text { Costa }(\%)\end{array}$ & 0,025 & 5 & Muito Alta \\
\hline $\begin{array}{l}\text { Mudanças } \\
\text { Relativas do } \\
\text { Nível do Mar } \\
\text { (mm/ano) }\end{array}$ & 12 & 2 & Muito Alta \\
\hline $\begin{array}{l}\text { Altura Signi- } \\
\text { ficativa da } \\
\text { Onda (m) }\end{array}$ & $1-2$ & 5 & Muito Alta \\
\hline $\begin{array}{l}\text { Amplitude de } \\
\text { Maré }(\mathrm{m})\end{array}$ & 0,5 & 5 & Muito Alta \\
\hline
\end{tabular}

$\mathrm{IVC}=\sqrt{\frac{\left(5^{*} 5^{*} 5^{*} 5^{*} * 5\right)}{6}} \cong 51,03$
Com relação às Taxas de Erosão/Acreção da Linha de Costa, estudos de Dillenburg et al. (2000); Martinho et al. (2009) concluíram que, o volume de sedimentos transportados ao longo da costa, bem como a energia de ondas, tende a aumentar conforme a costa torna-se convexa (projeções) e diminuir conforme a costa torna-se côncava (embaiamentos). A causa desse padrão está provavelmente relacionada aos processos de refração e dissipação, que as ondas de águas profundas encontram ao se aproximarem da costa. Quando a costa é côncava, a plataforma interna é larga e suave, esses processos ocorrem lentamente, e ao atingirem a costa, as ondas já perderam grande parte da sua energia. Assim, a deposição é favorecida. Por outro lado, quando a costa é convexa, a plataforma interna é estreita e íngreme, os processos de refração e dissipação ocorrem menos, e as ondas atingem a costa com alta energia. Portanto, o volume de sedimentos retirado destes locais é maior.

Somada à dinâmica natural, historicamente, a linha de costa adjacente a Praia do Cassino, vem sofrendo uma acreção relevante. Parte desta acreção se deve ao período que sucede a construção dos molhes da Barra do Rio Grande, a qual interferiu no balanço sedimentar da área, ao bloquear o transporte de sedimentos para nordeste, intensificando a acreção e acarretando um aumento localizado na largura da praia. Dessa forma, com uma acreção da linha de costa atual de aproximadamente 3,2m/ano (Lélis \& Calliari, 2004), a região está inserida num setor de barreira progradante. Porém, considerando o cenário de elevação acelerada do nível do mar prevista para os próximos anos, esta poderá se tornar retrogradante, com taxas de erosão da linha de costa estimadas em $11,47 \mathrm{~m} /$ ano no pior cenário projetado (com 10\% de probabilidade) para 2100 (Figueiredo, 2013).

Com relação às Mudanças Relativas do Nível do Mar, a região de estudo possui uma taxa atual de $2 \mathrm{~mm} / \mathrm{ano}$ de mudança relativa do nível do mar. Observa-se que o nível atual sobe lentamente, colocando a região costeira em um cenário de baixa vulnerabilidade. No entanto, quando consideradas as taxas para $2100(1,1 \mathrm{~m} / 90$ anos $)$, percebemos que a vulnerabilidade é considerada muito alta, quando o nível do mar atinge taxas de aproximadamente $12 \mathrm{~mm} / \mathrm{ano}$.

Através do exposto, percebe-se que, alterando os valores de somente duas variáveis, a Praia do Cassino deixaria de ser caracterizada como uma região moderadamente vulnerável, atualmente, e passaria a ser considerada como uma praia de vulnerabilidade muito alta às elevações do nível do mar, apresentando um IVC futuro de 51,03 .

\section{Conclusões}

A região costeira do Estado do Rio Grande do Sul, na qual a Praia do Cassino está inserida, possui caracterís- 
ticas geomorfológicas herdadas que, de forma geral, a torna bastante vulnerável às variações do nível médio do mar. Considerando o atual cenário de mudanças climáticas globais, é de extrema importância o estudo detalhado da área citada. Este se deu no presente estudo de forma qualitativa e quantitativa, de modo a determinar a vulnerabilidade costeira da região em questão, frente ao aspecto acima mencionado, com base nos resultados obtidos por Figueiredo (2013).

Neste contexto, como principais conclusões do trabalho efetuado destacam-se as seguintes:

- Conforme os cálculos de perda de área, considerando as prováveis futuras linhas de costa projetadas para 2100 , todos os ambientes da região de estudo apresentaram um percentual perdido de ambientes costeiros emersos, mesmo no melhor cenário considerado;

- Campos litorâneos e dunas representaram os maiores percentuais absolutos de perda ambiental quando considerado o pior cenário projetado (linha de costa modelada com $10 \%$ de probabilidade de ser ultrapassada em 2100);

- Sangradouros tiveram a totalidade de sua área perdida, mesmo no melhor cenário projetado (linha de costa modelada com $70 \%$ de probabilidade de ser ultrapassada em 2100);

- Os valores obtidos nos cálculos de IVC indicam que, a Praia do Cassino é classificada atualmente como moderadamente vulnerável às elevações do nível do mar, enquanto que nas situações projetadas para 2100 (Figueiredo, 2013), a área de estudo seria caracterizada como muito altamente vulnerável à subida do nível do mar.

Os dados obtidos na pesquisa são considerados extremamente úteis para a realização de adaptações na região de estudo, como obras de contenção ao avanço marinho, bem como de um manejo minucioso dos ambientes nela encontrados, no cenário de mudanças climáticas globais. Assim, será possível planejar adequadamente o uso, a ocupação e a adaptação desta região costeira, valendo-se, sobretudo, das individualidades de cada setor da costa do Rio Grande do Sul.

\section{Agradecimentos}

Agradecemos aos revisores deste artigo; à Coordenação de Aperfeiçoamento de Pessoal de Nível Superior (CAPES); a Agência Nacional do Petróleo (ANP), a qual subsidiou o trabalho original, base para este artigo; e a todas as pessoas e instituições que contribuíram para as experiências e aprendizado em gestão costeira.

\section{References}

Andrade, E.S.M.; Lacerda, G.B.M. (2009) - Mudanças Climáticas e Análise de Risco da Indústria de Petróleo no Litoral Brasileiro. 122p., Fundación Mapfre, Madrid, Espanha. ISBN: 9788498441642. Disponível on-line em http://www.mapfre.com/ documentacion/publico/118n/catalogo_imagenes/grupo.cmd?path=1053958.
Bindoff, N.L.; Willebrand, J.; Artale, V.; Cazenave, A.; Gregory, J.; Gulev, S.; Hanawa, K.; Le Quéré, C.; Levitus, S.; Nojiri, Y.; Shum, C.K.; Talley, L.D.; Unnikrishnan, A. (2007) - Observations: Oceanic Climate Change and Sea Level. In: Solomon, S.; Qin, D.; Manning, M.; Chen, Z.; Marquis, M.; Averyt, K.B.; Tignor, M.; Miller, H.L. (org.), Climate Change 2007: The Physical Science Basis. Contribution of Working Group I to the Fourth Assessment Report of the Intergovernmental Panel on Climate Change, pp.385-432, Cambridge University Press, Cambridge, U.K. / New York, NY, U.S.A. ISBN: 9780521705967. Disponível on-line em http://www.ipcc.ch/pdf/ assessment-report/ar4/wg1/ar4-wg1-chapter5.pdf

Bruun, P. (1988) - The Bruun Rule of erosion by sea-level rise - a discussion on large-scale two-dimensional and 3-dimensional usages. Journal of Coastal Research (ISSN: 0749-0208), 4(4):627-648, Coastal Education \& Research Foundation, Charlottesville, VA, U.S.A. Article Stable URL: http://www.jstor.org/stable/4297466

Carter, R.W.G. (1990) - Coastal Environments: An Introduction to the Physical, Ecological and Cultural systems of coastlines. 617p., Academic Press, San Diego, CA, U.S.A. ISBN: 9780121618568.

Calliari, L.J.; Klein, A.H.F. (1993) - Características morfodinâmicas e sedimentológicas das praias oceânicas entre Cassino e Chuí, RS. Pesquisas (ISSN: 0100-5375), 20(1):48-56, Instituto de Geociências, UFRGS, Porto Alegre, RS, Brasil. Disponível on-line em http://www.praia.log.furg.br/Publicacoes /1993/1993a.pdf

Church, J.A.; White, N.J. (2006) - A 20th century acceleration in global sea-level rise. Geophysical Research Letters, 33(1):1-4. DOI: $10.1029 / 2005$ GL024826

Church, J.A.; Clark, P.U.; Cazenave, A.; Gregory, J.M.; Jevrejeva, S.; Levermann, A.; Merrifield, M.A.; Milne, G.A.; Nerem, R.S.; Nunn, P.D.; Payne, A.J.; Pfeffer, W.T.; Stammer, D.; Unnikrishnan, A.S. (2013) - Sea Level Change. In: Stocker, T.F.; Qin, D.; Plattner, G.-K.; Tignor, M.; Allen, S.K.; Boschung, J.; Nauels, A.; Xia, Y.; Bex, V.; Midgley, P.M. (org.), Climate Change 2013: The Physical Science Basis. Contribution of Working Group I to the Fifth Assessment Report of the Intergovernmental Panel on Climate Change, pp.11371216. Cambridge University Press, Cambridge, United Kingdom, New York, NY, USA. ISBN: 978-1107057991. Disponível on-line em http://www.ipcc.ch/pdf/assessment-report/ar5/wg1/WG1AR5 _Chapter13_FINAL.pdf

Costa, C.S.B (1998) - Plantas de marismas e terras alagáveis. In: Seeliger, U.; Odebrecht, C.; Castello, J.P. (org.), Os Ecossistemas Costeiro e Marinho do Extremo Sul do Brasil, pp.2529, Editora Ecoscientia, Rio Grande, RS, Brasil. ISBN: 8587167014. Disponível on-line em http://www2.furg.br/instituto /io/ecoveco/ecomidia/livros/Os Ecossistemas Costeiro e Marinho do Extremo Sul do Brasil.pdf

Cowell, P.J.; Roy, P.S.; Jones, R.A. (1992) - Shoreface translation model: computer simulation of coastal-sand-body response to sea level rise. Mathematics and Computers in Simulation, 33(56):603-608. DOI: 10.1016/0378-4754(92)90158-D

Cowell, P.J.; Thom, B.G.; Jones, R.A.; Everts,C.H.; Simanovic, D. (2006). Management of Uncertainty in Predicting Climatechange Impacts on Beaches. Journal of Coastal Research 22(1):232-245. DOI: 10.2112/05A-0018.1

Diehl, F.P.; Spinola, J.L.; De Azevedo, N.T. (2010) - Mudanças climáticas globais e os impactos físicos e biológicos na zona costeira: Uma proposta de classificação. In: Dantas, M.B.; Séguin, E.; Ahmed, F. (org.), O Direito Ambiental na atualidade, pp.127-134,Lumen Juris Ltda., Rio de Janeiro, RJ, Brasil. ISBN: 9788537507087. Disponível on-line em http://www. ambito-juridico.com.br/site/index.php?n_link=revista_artigos_leitura\& artigo_id $=9162$ 
Dillenburg, S.R.; Roy, P.S.; Cowell, P.J.; Tomazelli, L.J. (2000) Influence of antecedent topography on coastal evolution as tested by the shoreface translation-barrier model (STM). Journal of Coastal Research (ISSN: 0749-0208), 16(1): 71-81, Coastal Education \& Research Foundation, Charlottesville, VA, U.S.A. Article Stable URL: http://www.jstor.org/stable/4300012

Dillenburg, S.R.; Tomazelli, L.J.; Martins, L.R.; Barbosa, E.G. (2005) - Modificações de longo período da linha de costa das barreiras costeiras do Rio Grande do Sul. Gravel (ISSN: 16785975), 3(1):9-14, Instituto de Geociências, UFRGS, Porto Alegre, RS, Brasil. Disponível on-line em http://www.ufrgs.br/gravel/3/Gravel_3_02.pdf

Figueiredo, S.A. (2013) - Modelling climate change effects in southern Brazil. Journal of Coastal Research, SI (65):19331938. DOI: $10.2112 /$ SI65-327.1

Figueiredo, S.A.; Calliari, L.J. (2005) - Sangradouros: Distribuição espacial, variação sazonal, padrões morfológicos e implicações no gerenciamento costeiro. Gravel (ISSN: 1678-5975), 3(1):4757, Instituto de Geociências, UFRGS, Porto Alegre, RS, Brasil. Disponível on-line em http://www.ufrgs.br/gravel/3/Gravel_3_06.pdf

Figueiredo, S.A. (2011) - External forcing and internal controls on coastal response to climate change at Rio Grande do Sul, southern Brazil. 164p., Tese de Doutorado, Universidade de Sidnei, Sidnei, Austrália. Disponível on-line em http://www.praia log.furg.br/Publicacoes/2011/2011b.pdf

Fontoura, J.A.S.; Almeida L. E.; Calliari, L. J.; Cavalcanti, A. M.; Möller, O. Jr., Romeu, M.A.R. and Christófaro, B.R. (2013) Coastal Hydrodynamics and Longshore Transport of Sand on Cassino Beach and on Mar Grosso Beach, Southern Brazil. Journal of Coastal Research, (4):855-869. DOI: 10.2112/JCOASTRES-D-11-00236.1

Gornitz, V. (1991) - Global sea level hazards from future sea level rise. Palaeogeography, Palaeoclimatology, Palaeoecology (Global and Planetary Change Section), 89(1):379-398. Elsevier Science Publishers B.V., Amsterdam, The Netherlands. Disponível on-line em http://pubs.giss.nasa.gov/docs/1991/ 1991_Gornitz 1.pdf

Hesp, P.A.; Dillenburg, S.R.; Barbosa, E.G.; Clerot, L.C.P.; Tomazelli, L.J.; Ayup-Zouain, R.N. (2007) - Morphology of the Itapeva to Tramandai transgressive dune field barrier system and mid-to late Holocene sea level change. Earth Surface Processes and Landforms, 32(3):407-414. DOI: 10.1002/esp.1408

Huiskes, A.H.L. (1990) - Possible effects of sea level changes on salt-marsh vegetation. In: Beukema, J. J.; J. J. W. M. Brouns (Eds.), Expected effects of climatic change on Marine Coastal Ecosystems, pp.167-172. Springer Netherlands, Netherlands. ISBN: 978-9400920033. Disponível on-line em 10.1007/97894-009-2003-3 20

IPCC (2001) - Climate Change 2001: The scientific basis. (Contribution of Working Group I to the Third Assessment Report of the Intergovernmental Panel on Climate Change). [Houghton, J.T., Y. Ding, D.J. Griggs, M. Noguer, P.J. van der Linden, X. Dai, K. Maskell, and C.A. Johnson (eds.)]. 873p., Cambridge University Press, Cambridge, United Kingdom / New York, NY, U.S.A. ISBN: 0521014956. Disponivel on-line em http://www.ipcc.ch/ipccreports/tar/wg1/pdf/WG1_TAR-FRONT.PDF

IPCC (2007) - Climate Change 2007: The physical science basis. (Contribution of Working Group I to the Fourth Assessment Report of the Intergovernmental Panel on Climate Change). [Solomon, S., D. Qin, M. Manning, Z. Chen, M. Marquis, K.B. Averyt, M.Tignor and H.L. Miller (eds.)]. 996 p., Cambridge University Press, Cambridge, United Kingdom and New York, NY, USA. Disponível on-line em http://www.ipcc.ch/pdf/assessmentreport/ar4/wg1/ar4_wg1_full_report.pdf
Lélis, R.J.F.; Calliari, L.J. (2004) - Historical shoreline changes near lagoonal and river stabilized inlets in Rio Grande do Sul State, southern Brazil. Journal of Coastal Research (ISSN: 07490208), SI(39): 301-305, Coastal Education \& Research Foundation, Charlottesville, VA, U.S.A. Disponível on-line em http://www.cerf-jcr.org/images/stories/59_lelis.pdf

Marengo, J.A. (2006). Mudanças Climáticas Globais e Efeitos sobre a Biodiversidade - Caracterização do clima atual e definição das alterações climáticas para o território Brasileiro ao longo do Século XXI. Subprojeto do Programa de Conservação e Utilização Sustentável da Diversidade Biológica Brasileira (PROBIO). 212 p., Instituto Brasileiro do Meio Ambiente e dos Recursos Naturais Renováveis, Brasília, DF, Brasil. ISBN: 8577380386. Disponível em http://www.mma.gov.br/estruturas/imprensa/ _arquivos/livro completo.pdf

Martin L, Flexor J-M, Vilas Boas GS, Bittencourt ACSP, Guimaraes MMM. (1979). Courbe de variations du niveau relative de la mer aucours des 7000 dernieres annees sur un secteur homogene du littoral bresilien (nord de Salvador, Bahia). In: Sugio K.; Fairchild R.R.; Martin L.; Flexor J.M. (Eds.) Proceedings of the International Symposium on Coastal Evolution in the Quaternary, pp.264 -274, Editora da Universidade de Sao Paulo, São Paulo, SP, Brasil. ISBN: 978-8531405297. Disponível on-line em http://horizon.documentation.ird.fr/exl-doc/pleins textes/pleins textes 6 /b_fdi_35-36/41921.pdf

Martinho, C.T.; Dillenburg, S.R.; Hesp, P. (2009) - Wave energy and longshore sediment transport gradientes controlling barrier evolution in Rio Grande do Sul, Brazil. Journal of Coastal Research, 25(2):285-293. DOI: 10.2112/06-0645.1

Meehl, G.A.; Stocker, T.F.; Collins, W.D.; Friedlingstein, P.; Gaye, A.T.; Gregory, J.M.; Kitoh, A.; Knutti, R.; Murphy, J.M.; Noda, A.; Raper, S.C.B.; Watterson, I.G.; Weaver, A.J.; Zhao, Z.-C. (2007) - Global Climate Projections. In: Solomon, S.; Qin, D.; Manning, M.; Chen, Z.; Marquis, M.; Averyt, K.B.; Tignor, M.; Miller, H.L. (org.), Climate Change 2007: The Physical Science Basis. Contribution of Working Group I to the Fourth Assessment Report of the Intergovernmental Panel on Climate Change, pp. 749-844, Cambridge University Press, Cambridge, U.K. / New York, NY, U.S.A. ISBN: 978-0521705967. Disponível on-line em http://www.ipcc.ch/pdf/assessmentreport/ar4/wg1/ar4-wg1-chapter10.pdf

Mesquita, A. R. (2003) - Sea level variations along the Brazilian coast: a short review. Journal of Coastal Research, (ISSN: 0749-0208), SI(35): 21-31, Coastal Education \& Research Foundation, Charlottesville, VA, U.S.A. Article Stable URL: http://www.jstor.org/stable/40928745

Pianca, C.; Mazzini, P. L. F.; Siegle, E. (2010) - Brazilian offshore wave climate based on NWW3 reanalysis. Brazilian Journal of Oceanography, 58(1):53-70. DOI: 10.1590/S167987592010000100006

Rahmstorf, S. (2007) - A semi-empirical approach to projecting future sea-level rise. Science, 315(5810):368-370. DOI: 10.1126/science. 1135456

Toldo Jr., E. E.; Almeida, L.E.S.B.; Nicolodi, J.L.; Martins, L.R. (2005) - Retração e progradação da zona costeira do estado do Rio Grande do Sul. Gravel (ISSN: 1678-5975), 3(1): 31-38. Instituto de Geociências, UFRGS, Porto Alegre, RS, Brasil. Disponível on-line em http://www.ufrgs.br/gravel/3/Gravel_3_04.pdf

Tomazelli, L.J.; Villwock, J.A. (2005) - Mapeamento geológico de planícies costeiras: o exemplo da costa do Rio Grande do Sul. Gravel (ISSN: 1678-5975),3(1):109-115. Instituto de Geociências, UFRGS, Porto Alegre, RS, Brasil. Disponível on-line em http://www.ufrgs.br/gravel/3/Gravel_3_11.pdf 